\title{
Nod2 deficiency is associated with an increased mucosal immunoregulatory response to commensal microorganisms
}

\author{
A Amendola ${ }^{1,4}$, A Butera ${ }^{1,4}, M_{\text {Sanchez }}^{2}$, W Strober ${ }^{3}$ and $M$ Boirivant $^{1}$
}

On the basis of previous studies demonstrating that a breach of the colonic epithelial barrier is associated with a microbiota-dependent increase in lamina propria (LP) regulatory cells, we investigated if the lack of spontaneous intestinal inflammation observed in nucleotide-binding oligomerization domain 2 (Nod2) - / - mice was due to enhanced intestinal regulatory function. We found that the LP CD4 + T-cell population of Nod2 - I- mice contains an increased percentage of CD4 + regulatory T cells bearing transforming growth factor $-\beta /$ latency peptide (LP CD4 + LAP (latency-associated peptide) + T cells) both under baseline conditions and following an intentional breach of the colonic barrier induced by ethanol administration. In addition, we found that Nod $2-/$ - mice manifest decreased severity of 2,4,6-trinitrobenzene sulfonic acid (TNBS)-colitis and that TNBS-colitis in Nod2 - / - or $\mathrm{Nod} 2+I+$ mice is ameliorated by adoptive transfer of LP cells from ethanol-treated mice before, but not after, depletion of LAP + T cells. This increased regulatory T-cell response in Nod $2-/$ - mice could explain why NOD2 polymorphisms in humans are not in themselves sufficient to establish inflammatory lesions.

\section{INTRODUCTION}

NOD2 (nucleotide-binding oligomerization domain 2) is a member of the NLR (NOD, leucine-rich repeat-containing protein) family of intracellular microbial sensors that has gained prominence, because polymorphisms in the CARD15 gene encoding this protein is the single most important genetic risk factor in Crohn's disease. ${ }^{1-4}$ The NOD2 leucine-rich repeat sensor recognizes muramyl dipeptide, a component of the peptidoglycan present in the bacterial cell wall and thus NOD2 is likely to be an innate immune factor that participates in the control of organisms that enter the lamina propria (LP). This has led to the view that abnormal Nod 2 function associated with leucine-rich repeat polymorphisms leads to blunted clearance of such organisms and thus an inflammatory response mediated by innate immune functions unrelated to Nod2. ${ }^{5,6}$ However, another view is based on evidence that Nod2 is a negative regulator of Toll-like receptor (TLR) signaling and its deficiency results in enhanced production of
Th1-polarizing cytokines in the TLR-rich gut micro-environment. ${ }^{7}$ Mice with Nod2 deficiency have characteristics that bear on this question. For instance it has been shown that Nod2 - / mice exhibit increased CD4 + T-cell interferon (IFN) $\gamma$ production that is dependent on the presence of the intestinal microbiota and this, in turn, leads to increased bacterial translocation into the Peyer's patches and increased Peyer's patch epithelial permeability due to induction of myosin-light chain kinase (MLCK), a factor that downregulates tight junction integrity. Furthermore, such T cell-epithelial cell cross-talk seems to be under the control of TLR signaling, which is increased in Nod2-/ - mice, but can be downregulated in Nod $2+/+$ mice by administration of muramyl dipeptide (Nod2 ligand). Thus, it appears that bacterial translocation in Nod2 - / - mice results from an absence of Nod2 regulation of TLR function. ${ }^{8,9}$ These findings favor the second hypothesis relating to polymorphic NOD2 in Crohn's disease, namely that the latter leads to hyper-responsiveness. ${ }^{10}$

\footnotetext{
${ }^{1}$ Immune-Mediated Diseases Section, Department of Infectious, Parasitic, and Immune-Mediated Diseases, Rome, Italy. ${ }^{2}$ Department of Cell Biology and Neurosciences, Istituto Superiore di Sanità, Rome, Italy and ${ }^{3}$ Mucosal Immunity Section, Laboratory of Host Defenses, National Institute of Allergy and Infectious Diseases, NIH, Bethesda, Maryland, USA. Correspondence: M Boirivant (monica.boirivant@iss.it)

${ }^{4}$ The first two authors contributed equally to this work.
} 
Despite the above mentioned permeability changes, Nod2 - / - mice do not develop overt intestinal inflammation, suggesting that Nod2 abnormalities are not sufficient to cause spontaneous and full-blown inflammatory lesions in themselves. Possibly relating to this, we previously demonstrated that a transient breach of the colonic epithelial barrier and an associated transient increase in the intestinal permeability is characterized by a microbiota-dependent increase in the generation of regulatory cytokines and cells. In particular, such breaches were associated with the expansion Foxp3negative $\mathrm{CD} 4+\mathrm{T}$ cells expressing surface transforming growth factor beta (TGF- $\beta$ ) associated with the latency-associated peptide (LAP) $(\mathrm{CD} 4+\mathrm{LAP}+\mathrm{T}$ cells) that render mice resistant to the induction of 2,4,6-trinitrobenzene sulfonic acid (TNBS)-induced colitis. ${ }^{11}$ Thus, the lack of spontaneous inflammation in mice with Nod2 deficiency may be due to an enhanced mucosal regulatory response. To explore this hypothesis, we investigated the mucosal regulatory response of mice with Nod2 deficiency following a breach of the colonic barrier. We found that the LP of Nod2-/ - mice, when compared with $\mathrm{Nod} 2+/+$ (wild-type) mice, contains an increased percentage $\mathrm{CD} 4+\mathrm{T}$ cells that are $\mathrm{CD} 4+\mathrm{LAP}+$ regulatory $\mathrm{T}$ cells; furthermore, we found using cell transfer studies that these regulatory cells are likely to be responsible for the decreased severity of TNBS-colitis observable in Nod2 - / - mice. Thus, an increased regulatory T-cell response to microbiota in Nod2 - / - mice could indeed explain why NOD2 polymorphisms in humans are not sufficient to establish inflammatory lesions in the absence of other abnormalities.

\section{RESULTS \\ Nod2 - I - mice exhibit increased colonic permeability associated with an expanded subpopulation of LP CD4 + LAP + T cells}

As it has been reported that Nod2 $-/-$ mice show increased Peyer's patch permeability and bacterial translocation, ${ }^{8}$ in initial studies we assessed colonic permeability and in vivo cytokine production in untreated Nod2 $-/-$ mice. As shown in Figure 1, we found that Nod2 - / - mice exhibit a significant increase in baseline colonic permeability when compared with $\mathrm{Nod} 2+/+$ mice as measured by uptake of intrarectal (i.r.) fluorescein isothiocyanate (FITC)-dextran into the serum. Moreover, as also shown in Figure 1, this permeability change was associated with a significant increase in in vivo interleukin (IL)-12p70 production as measured by LP tissue content of this cytokine (as well as tumor necrosis factor- $\alpha$, data not shown), suggesting that the permeability change had led to increased stimulation of LP macrophages and/or dendritic cells (DCs) by innate stimuli derived from the intestinal microbiota. One might predict that this increased IL-12p70 production would be accompanied by increased LP T-cell IFN- $\gamma$ production, but, as shown in Figure 1, in vivo IFN- $\gamma$ production in Nod $2-/-$ mice was not increased as assessed by measurement of tissue content of this cytokine. It should be noted, however, that Nod2 - / - LP T cells showed some evidence of enhanced Th1 differentiation in that stimulation of purified LP mononuclear cell (LPMC) with anti-CD3/CD28 exhibited a significant increase in IFN- $\gamma$ production in vitro.

To examine if the above lack of IFN- $\gamma$ production in vivo could be due to downregulation of LP T-cell production of this cytokine we next determined if the Nod2-/ - LP contained increased regulatory factors. As shown in Figure 1, panel e, flow cytometric studies revealed that Nod2 - / - LP CD4 + T-cell populations contained a significantly increased percentage of LP $\mathrm{CD} 4+\mathrm{LAP}+\mathrm{T}$ cells and, moreover, Nod2 - / - mice LP exhibited a significant increase in tissue content of TGF- $\beta$, as compared with cells and tissues, respectively, from $\mathrm{Nod} 2+/+$ mice (Figure 1, panel f). However, we did not observe an increase in the percentage of $\mathrm{CD} 4+$ Foxp $3+\mathrm{T}$ cells within the LP CD $4+$ T-cell population (Nod2-/ - : $11.33 \% \pm 1.42$ vs. Nod $2+/+: 10.68 \% \pm 0.71$; mean \pm s.e.; $P=0.65$ ). The above findings thus provide initial support for the possibility that the lack of increased IFN- $\gamma$ secretion in vivo in Nod $2-/-$ mice is in fact due to downregulation of pro-inflammatory T-cell differentiation by regulatory cells that do not express Foxp3.

Enhanced expression of CD4 + LAP + T Cells in Nod2 - I mice is dependent on an intact intestinal microbiota

In previous studies, it was shown that the intestinal microbiota acting through myosin light chain kinase (MLCK)-mediated effects on epithelial tight junctions was responsible for changes in the permeability of the Peyer's patch epithelium in Nod $2-/-$ mice. ${ }^{9}$ We reasoned that a similar mechanism may obtain with respect to the increased numbers of LP $\mathrm{CD} 4+\mathrm{LAP}+\mathrm{T}$ cells observed in Nod $2-/-$ mice inasmuch as we have shown previously that induction of increased numbers of the latter cells in wild-type mice is also dependent on the presence of an intact intestinal microbiota. ${ }^{11}$ In initial studies to examine this question we treated mice with ampicillin for 3 weeks and evaluated the frequency of LP CD4 + LAP + $\mathrm{T}$ cells in the treated mice (as well as in the untreated control mice). As shown in Figure 2, panel a, although ampicillin administration to Nod $2+/+$ mice did not significantly affect the percentage of $\mathrm{LP} \mathrm{CD} 4+\mathrm{LAP}+\mathrm{T}$ cells, administration to Nod2 - / - mice was associated with a significant reduction of the percentage of LP CD4 + LAP + cells and at the end of the treatment period LP CD4 + LAP + levels in Nod $2-/-$ mice were comparable to those in Nod $2+/+$ mice. Thus, the increased numbers of LP CD4 + LAP $+\mathrm{T}$ cells in Nod $2-/-$ mice is dependent on the presence of an intact intestinal microbiota.

With this information in hand, we next turned our attention to role of MLCK in the expansion of LP CD4 + LAP + T cells in Nod $2-/$ - mice. To this end, we determined LP CD $4+$ LAP + T-cell levels in Nod2 $-/-$ and Nod $2+/+$ mice pre-treated with an agent, ML-7, that has been shown to block the kinase activity of MLCK and thus inhibit its effect on intestinal permeability. ${ }^{9}$ In initial studies shown in Figure 2, panel b, we first verified this effect by showing that ML-7 administration led to a reduction in serum concentration of intrarectally administered FITC-dextran in both $\mathrm{Nod} 2-/-$ and Nod $2+/+$ mice. Then, as shown in Figure 2, panel c, we 
a

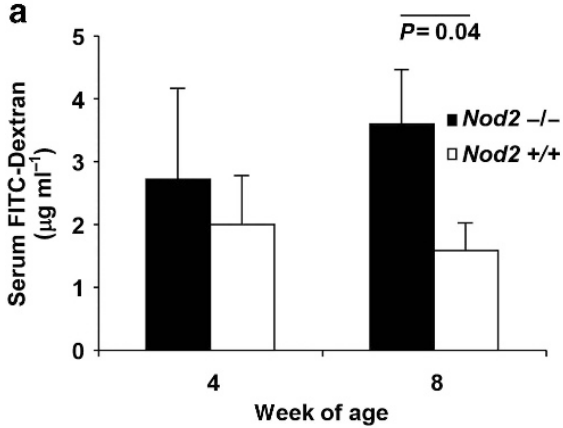

C

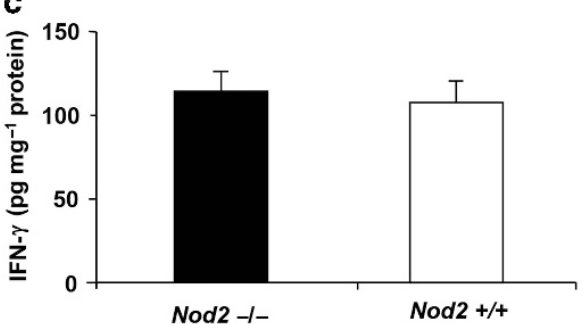

e

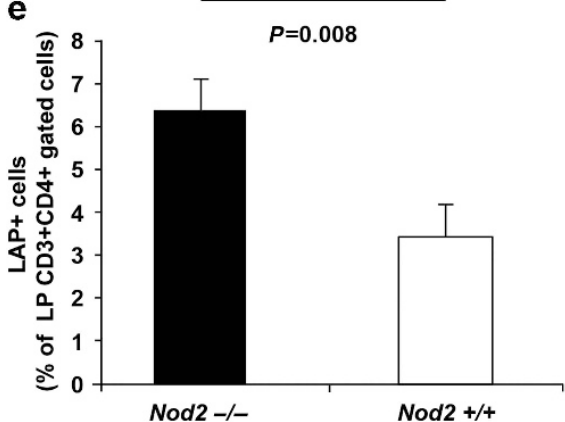

b
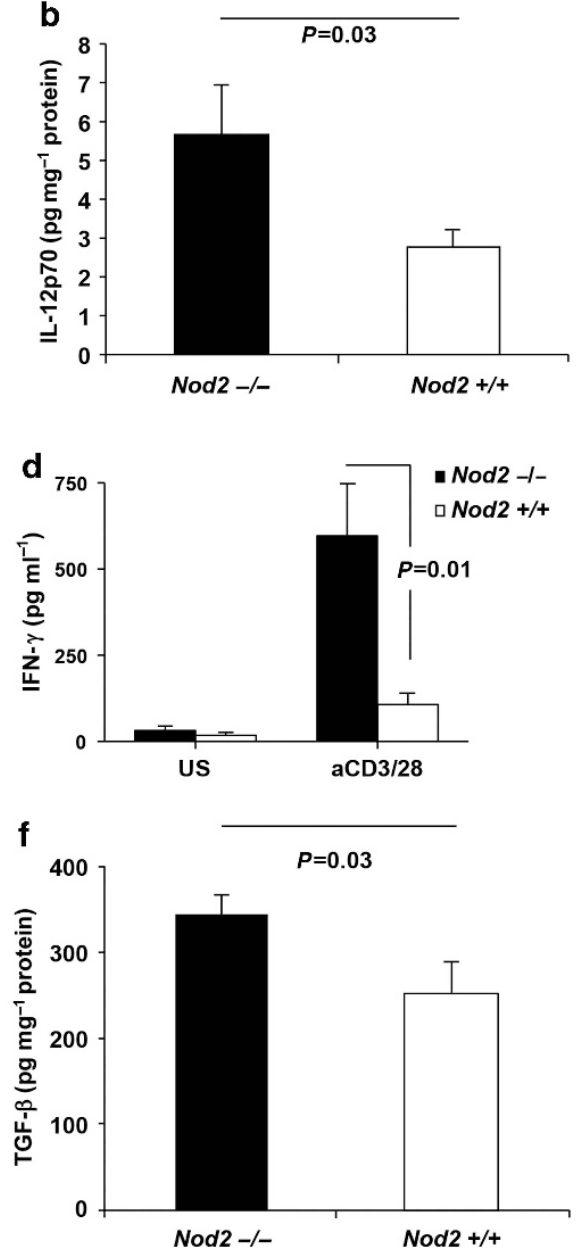

Figure 1 Nucleotide-binding oligomerization domain 2 (Nod2) - / - mice exhibit increased colonic permeability and increased in vivo interleukin (IL)12 p70 production, associated with an increased lamina propria (LP) subpopulation of CD4 + LAP (latency-associated peptide) + T cells and enhanced in vivo transforming growth factor beta (TGF- $\beta$ ) production. (a) measurement of colonic permeability by i.r. administration of fluorescein isothiocyanate (FITC)-Dextran (see Methods). Data shown are derived from two separate experiments, each involving five mice per group; data represent mean \pm s.e.; (b, c) In vivo interleukin (IL)-12p70 and interferon (IFN)- $\gamma$ production: cytokine content of protein extracted from lamina propria tissue was measured by enzyme-linked immunosorbent assay (ELISA); data represent mean \pm s.e. derived from 12 mice in each group; (d) lamina propria mononuclear cells (LPMCs) isolated from colonic tissue were cultured for $48 \mathrm{~h}$ in the absence of stimulation (unstimulated, US) or in the presence of anti-CD3/28 stimulation, after which the culture supernatants were assayed for IFN- $\gamma$ content by ELISA; data represent mean \pm s.e. derived from 10 mice in each group. Data were tested for statistical significance using Student's $t$-test. (e) Percentage of LAP + cells among CD3 + CD4 + gated LP cells; data represent mean \pm s.e. derived from 12 mice per group; (f) in vivo TGF- $\beta$ production: TGF- $\beta$ content of protein extracted from lamina propria tissue was measured by ELISA. Data represent mean \pm s.e. derived from 12 mice per group. Data were tested for statistical significance using Student's $t$-test.

showed that ML-7 administration led to a statistically significant reduction in the number of $\mathrm{LP} \mathrm{CD} 4+\mathrm{LAP}+\mathrm{T}$ cells in Nod $2-/-$ mice that was now not statistically different from that in Nod $2+/+$ mice. Taken together, these studies suggest that an intact intestinal microbiota is necessary for the induction of increased LP CD $4+\mathrm{LAP}+\mathrm{T}$ cells in Nod $2-/-$ mice and that such induction acts, at least in part, via MLCK effects on intestinal permeability.

\section{The role of CD11c + DCs in LP CD4 + LAP + T-cell expansion in Nod2 $-I-$ mice}

In previous studies, we showed that CD11c + DCs from ethanol-treated mice induced a greater increase in LP CD4+ $\mathrm{LAP}+\mathrm{T}$ cells among co-cultured CD4 $+\mathrm{T}$ cells than an identical population of DCs from untreated mice. This, taken with the observation that suppression of in vivo TLR2 responses by TLR 2 siRNA administration had a downregulatory effect on induction of LP CD4 + LAP + T cells, suggested that TLRstimulated $\mathrm{CD} 11 \mathrm{c}+\mathrm{DCs}$ were driving $\mathrm{LP} \mathrm{CD} 4+\mathrm{LAP}+$ T-cell expansion. ${ }^{11}$ Applying this information to a further examination of the role of CD11c + DCs in the increased of LP $\mathrm{CD} 4+\mathrm{LAP}+\mathrm{T}$ cells in Nod $2-/-$ mice, we co-cultured purified LP CD11c + cells from Nod $2-/-$ and Nod $2+/+$ with isolated LP CD $4+$ cells from Nod $2-/-$ and Nod $2+/+$ mice (at a 1:2 ratio) in various combinations and then assessed LAP + expression after $48 \mathrm{~h}$ of culture. As shown in Figure 3, panel a, co-culture of CD11c + DCs with CD $4+$ T cells from Nod2 $-/-$ mice was associated with a larger increase in $\mathrm{LAP}+\mathrm{T}$ cells as compared with the increase in LAP $+\mathrm{T}$ cells in co-cultures of CD11c + DCs and CD $4+\mathrm{T}$ cells from 
a

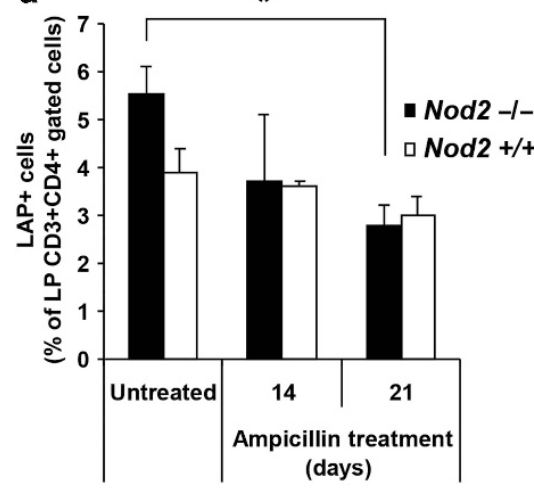

b

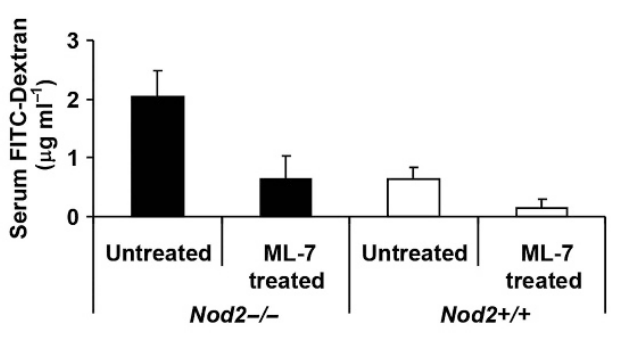

c

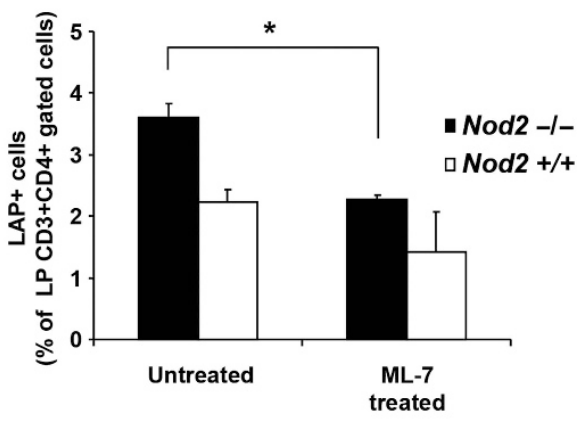

Figure 2 Effect of ampicillin treatment (a) and ML-7 treatment (b, $\mathbf{c})$ on the fraction of lamina propria (LP) CD4 + LAP (latency-associated peptide) + cells among CD4 + LP cells. (a) Separate groups of mice were treated with ampicillin $200 \mu \mathrm{g}$ per $100 \mu \mathrm{l}$ distilled water or $100 \mu \mathrm{l}$ distilled water alone (untreated) by oral gavage;for 14-21 days. Mice were killed at 14 and 21 days of ampicillin treatment, and at 21 days of water treatment, respectively. LP cells from colons isolated, stained for CD3, CD4 and LAP and analyzed by flow cytometry. Each column represent mean \pm s.e. ${ }^{*} P<0.05$. Nucleotidebinding oligomerization domain 2 (Nod2) - / - mice untreated (distilled water only) vs. ampicillin-treated 21 days by Student's $t$-test. (b) Separate groups of mice were treated for 4 days with ML-7 twice a day i.p (see Methods). At the end of treatment, mice were i.r. administered fluorescein isothiocyanate (FITC)-Dextran (see Methods). Columns represent mean \pm s.e. of three mice per group. (c) Separate groups of mice were treated for 4 days with ML-7 twice a day i.p (see Methods). At the end of treatment mice were sacrificed, colons were collected and isolated LP cells stained for CD3, CD4 and LAP and analyzed by flow cytometry. Columns represent mean \pm s.e. of data from three different experiments. ${ }^{\star} P<0.05$. Nod2 $-/-$ mice untreated vs. ML-7 treated mice by Student's $t$-test.
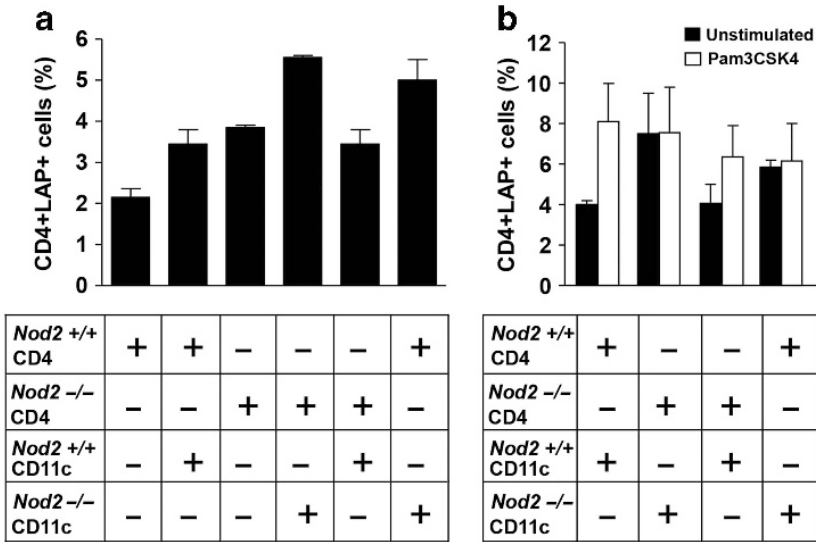

\begin{tabular}{|l|c|c|c|c|}
\hline $\begin{array}{l}\text { Nod2 +/+ } \\
\text { CD4 }\end{array}$ & + & - & - & + \\
\hline $\begin{array}{l}\text { Nod2 }-/- \\
\text { CD4 }\end{array}$ & - & + & + & - \\
\hline $\begin{array}{l}\text { Nod2 +/+ } \\
\text { CD11c }\end{array}$ & + & - & + & - \\
\hline $\begin{array}{l}\text { Nod2 -/- } \\
\text { CD11c }\end{array}$ & - & + & - & + \\
\hline
\end{tabular}

Figure 3 Induction of lamina propria (LP) CD4 + LAP (latencyassociated peptide) + cells by CD11c dendritic cells (DCs) in vitro. (a) Percentages of $\mathrm{LAP}^{+}$cells in purified lamina propria CD4 $+\mathrm{T}$-cell populations following co-culture for $48 \mathrm{~h}$ with $\mathrm{CD}_{11 \mathrm{c}^{+}} \mathrm{DCs}$ isolated from the lamina propria of nucleotide-binding oligomerization domain 2 (Nod2) $-/-$ and Nod2 $+/+$ mice. Column represent mean \pm s.e. of data from two different experiments. (b) Percentages of $\mathrm{LAP}^{+}$cells in purified lamina propria CD4 + T-cell populations following co-culture for $48 \mathrm{~h}$ with $\mathrm{CD} 11 \mathrm{C}^{+}$DCs isolated from the lamina propria of Nod2 $-/-$and $\mathrm{Nod} 2+I+$ mice in the presence or in the absence of Pam3CSK4. Column represent mean \pm s.e. of data from two different experiments.

Nod $2+/+$ mice. Furthermore, increases seen in co-cultures of CD11c + DCs from Nod $2-/-$ mice and CD $4+$ T cells were equal regardless of the source of CD4 $+\mathrm{T}$ cells. These data thus suggest that DCs from Nod $2-/-$ mice are more efficient in inducing the expansion of LAP + T cells as compared with DCs from $\mathrm{Nod} 2+/+$ mice.

In previous studies, we also showed that TLR2 stimulation was responsible for the increased in LP CD4 + LAP + cell level observed after ethanol-induced increase of epithelial permeability(11). We performed co-culture experiments in the presence of the TLR2 stimulant Pam3CSK4 to further investigate the role of LP DCs in LP CD4 $+\mathrm{LAP}+$ cell expression. As shown in Figure 3, panel b, TLR2 stimulation of CD11c cells from Nod2 $-/-$ mice co-cultured with CD4 + cells from either Nod2 $-/$ - or Nod2 $+/+$ mice did not increase the percentage of LP CD4 $+\mathrm{LAP}+$ cells over the already increased percentage observed in unstimulated co-cultures. In contrast, TLR2 stimulation of CD11c cells from $\mathrm{Nod} 2+/+$ mice co-cultured with CD4 + cells from Nod $2-/-$ or Nod $2+/+$ mice led to an increase in the percentage of LP CD4 + LAP + cells over the relatively low percentage observed in unstimulated co-cultures in two experiments.

Thus, CD11c DCs not subjected to "in vitro" TLR2 stimulation from Nod $2-/-$ mice are as efficient in inducing the expansion of LP CD4 + LAP $+\mathrm{T}$ cells due to sustained "in vivo" TLR2 stimulation as CD11c cells subjected to "in vitro" TLR2 stimulation from Nod $2+/+$ mice. In view of the dependence of LP CD4 + LAP + T cells on the intestinal microbiota, these data also suggest that DCs stimulated by TLR ligands in the intestinal microbiota are driving the increase in the number of these cells in the LP of Nod2-/ - mice.

\section{Nod2 - / - mice mount an enhanced regulatory T-cell response and exhibit less inflammation upon challenge with i.r. ethanol}

In a previous study, we showed that the number of LP CD4 + $\mathrm{LAP}+\mathrm{T}$ cells in the colonic mucosa was augmented by administration of an agent, such as ethanol, that increases the permeability of the mucosal epithelial barrier. This and the fact that Nod2-/- mice display increased baseline mucosal permeability prompted us to explore further the lack of inflammation in the LP of Nod2-/ - mice and its possible relation to the presence of increased numbers of LP CD4+ $\mathrm{LAP}+$ regulatory T cells. To this end, we conducted studies in which $\operatorname{Nod} 2-/-$ mice are subjected to intentional induction 


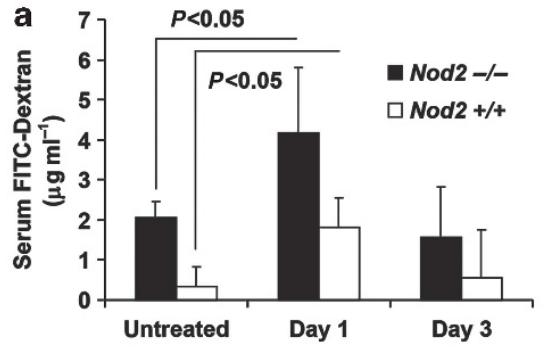

C
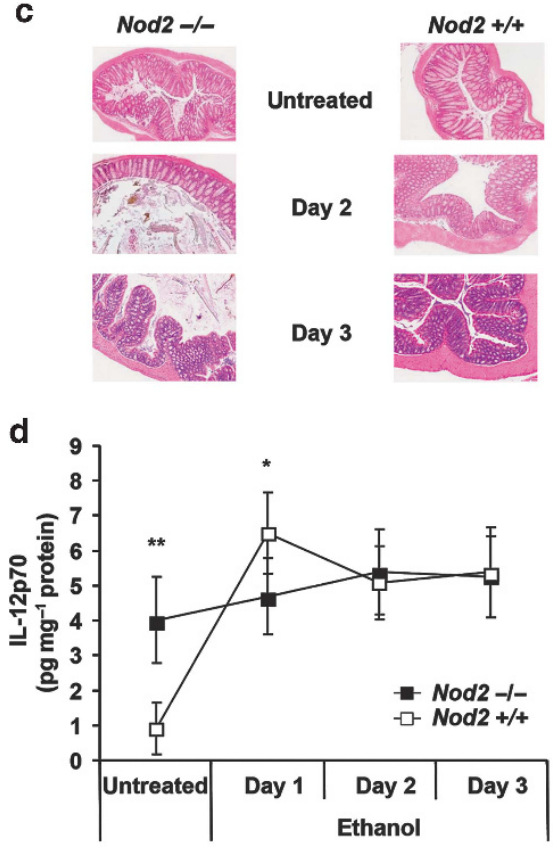

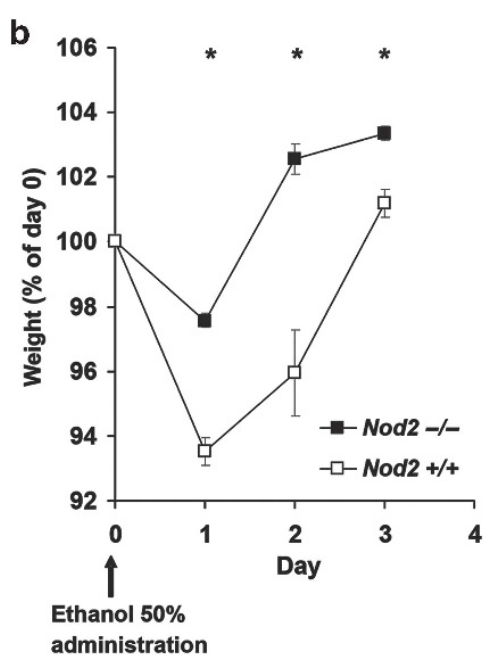

administration

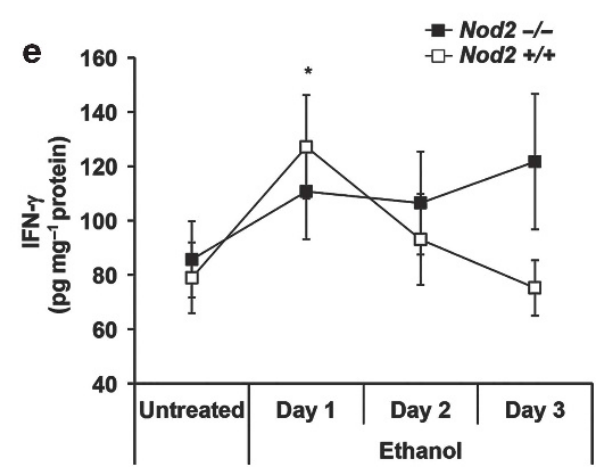

Figure 4 Nucleotide-binding oligomerization domain 2 (Nod2) $-/-$ mice exhibit increased permeability but decreased inflammation vs. Nod2 $+/+$ mice upon intrarectal ethanol administration. (a) Colonic permeability in Nod2 - / - and Nod2 $+/+$ mice after administration of intrarectal $50 \%$ ethanol measured by serum fluorescein isothiocyanate (FITC)-dextran uptake (See Methods); data represent mean \pm s.e. derived from two different experiments in which three mice per group were evaluated; ${ }^{*} P<0.05$ day 1 vs. untreated; (b) mouse body weight changes after intrarectal ethanol administration; each point represents mean \pm s.e. of daily weights expressed as percentage of day 0 weight; data represent pooled values derived from three different experiments in which five mice per group was studied. ${ }^{*} P<0.01$ Nod $2-/-$ vs. Nod2 $+/+$; (c) representative microscopic appearance of colons of mice administered ethanol and killed at the time points shown; hematoxylin and eosin (H\&E) staining original magnification $\times 40$. Nod2 $-/-$ and Nod2 $+/+$ untreated and day 3: normal colonic architecture/patterns. Nod2 $+/+$ day 2: presence of mild submucosal edema and mild inflammatory infiltration. Nod2 - /- day 2: normal colonic architecture/pattern; (d) in vivo interleukin (IL)-12p70 production after 50\% ethanol administration. Each point represents mean \pm s.e. of pooled values derived from two experiments in which five mice per group were evaluated at each time point; ${ }^{\star} P<0.05$ Nod $2+/+$ day 1 vs. untreated mice. ${ }^{* *} P<0.05$ Nod2 $-/-$ vs. Nod $2+/+$ untreated mice. (e) Interferon (IFN)- $\gamma$ tissue content at different days after ethanol administration. Each point represents mean \pm s.e. as described in panel $\mathbf{d}$; ${ }^{*} P<0.05$ Nod2 $+/+$ day 1 vs. untreated. Data were tested for statistical significance using Student's $t$-test.

of increased mucosal permeability by ethanol administration. As shown in Figure 4, panel a, i.r. ethanol administration caused a transient increase in colonic permeability in both Nod $2-/-$ and Nod $2+/+$ mice. As shown in Figure 4, panel b, despite such increased permeability, Nod $2-/-$ mice displayed significantly less weight loss as compared with $\mathrm{Nod} 2+/+$ mice and, as shown in Figure 4, panel c, Nod2 - / - mice exhibited little or no intestinal inflammation (colitis score: 0 ), whereas $\mathrm{Nod} 2+/+$ mice exhibited mild intestinal inflammation (colitis score: 1-2). In vivo IL-12p70 and IFN- $\gamma$ responses to ethanol administration provided parallel results; thus, as shown in Figure 4, panel d, although baseline IL-12p70 production was higher in Nod $2-/-$ mice than in Nod $2+/+$ mice, the latter mice exhibited a sharp rise in in vivo IL-12p70 production that led to a production level that exceeded that of Nod $2-/$ - mice on the day of maximal permeability change, whereas $\mathrm{Nod} 2-/$ - mice did not show any significant change in IL-12p70 tissue content after ethanol administration. As shown in Figure 4, panel e, a similar picture was observed for in vivo IFN- $\gamma$ production although in this case baseline production of this cytokine were essentially the same in Nod $2-/-$ and $\mathrm{Nod} 2+/+$ mice. Corroborative results were obtained when we administered a second dose of $50 \%$ ethanol to the mice on day 4 . Thus, as shown in Supplementary Figure 1 online, such secondary ethanol administration was again associated with less weight loss in Nod2 - / - as compared with Nod $2+/+$ mice.

The above difference between Nod $2-/-$ and Nod $2+/+$ mice in their response to i.r. ethanol administration could be the result from the fact that $\mathrm{Nod} 2-/-$ mice mount a greater 

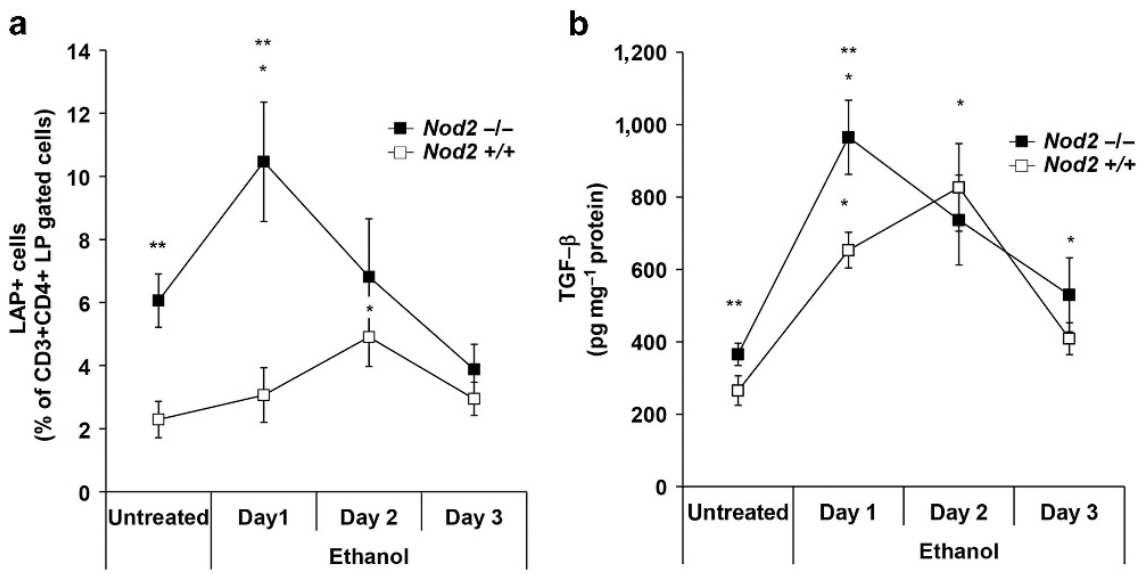

Figure 5 Effect of ethanol administration on CD4 + LAP (latency-associated peptide) + T cells. (a) Percentage of LAP + T cells among CD3 + CD4 + gated lamina propria $(\mathrm{LP})$ cells after ethanol administration. Each point represents mean \pm s.e. of pooled values derived from three experiments in which five mice per group were evaluated; ${ }^{*} P<0.05$ nucleotide-binding oligomerization domain 2 (Nod2) $-/-$ day 1 vs. untreated; ${ }^{*} P<0.05$ Nod $2+/+$ day 2 vs. untreated; ${ }^{* *} P<0.05$ Nod2 $-/$ - vs. Nod2 $+/+$ untreated and day 1; (b) In vivo transforming growth factor beta (TGF- $\beta$ ) production after ethanol administration. Each point represents mean \pm s.e. as described in panel a. ${ }^{*} P<0.05$ day $1-2$ vs. untreated Nod $2-/-$ mice; ${ }^{*} P<0.05$ day $1-3$ vs. untreated Nod2 $+/+$ mice; ${ }^{\star \star} P<0.05$ untreated and day 1 Nod $2-/-$ vs. Nod $2+/+$ mice. Data were tested for statistical significance using Student's $t$-test.

a

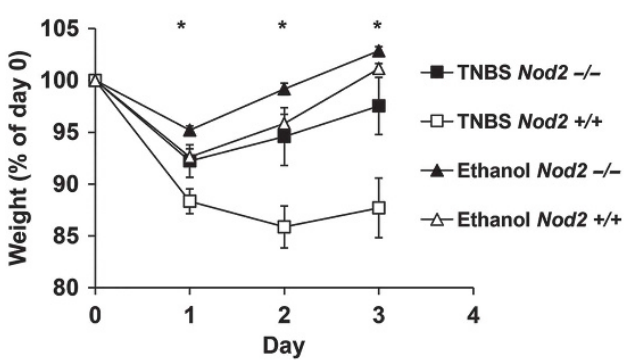

C

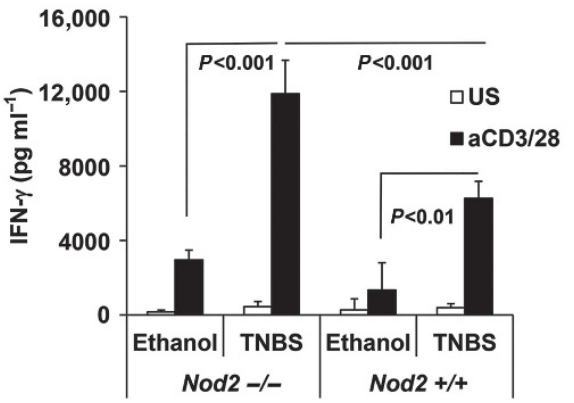

b

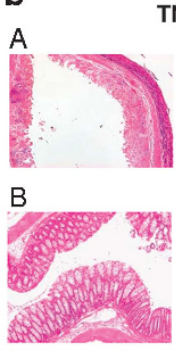

TNBS colitis

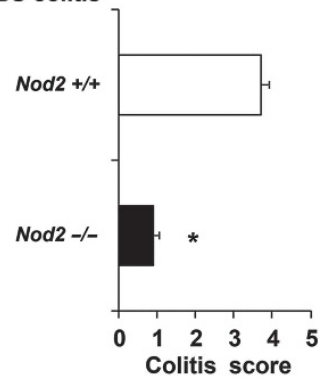

d

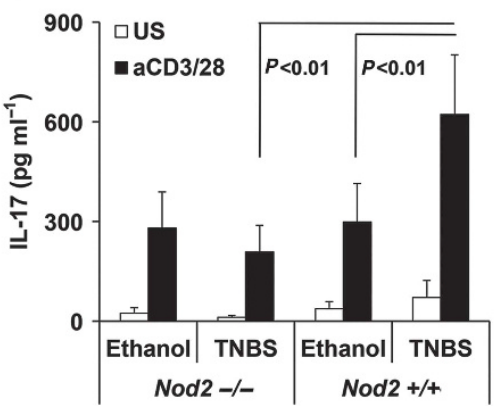

Figure 6 Nucleotide-binding oligomerization domain 2 (Nod2) - /- mice exhibit less severe 2,4,6-trinitrobenzene sulfonic acid (TNBS)-colitis compared with Nod2 $+/+$ mice. Mice were administered intrarectal ethanol/TNBS to induce TNBS-colitis or ethanol alone. (a) Body weight changes; each point represents mean \pm s.e. weight at indicated times expressed as percentage of day 0 weight and derived from three different experiments in which five mice per group were evaluated; ${ }^{*} P<0.05$ ethanol/TNBS-treated Nod $2-/-$ vs. Nod $2+/+;$ (b) histological appearance of representative colonic tissue of TNBS-treated mice killed at day 3 after TNBS administration; hematoxylin and eosin (H\&E) staining, original magnification $\times 40$ : (A) $\mathrm{Nod} 2+/+$ : severe, diffuse ulcerative colitis with dense transmural inflammatory cell infiltration of the submucosa, the smooth muscle and the serosa layers. (B) Nod2 - / - mild, non-ulcerative colitis with mild submucosal edema and mild inflammatory cell infiltration; Colitis score ${ }^{*} P<0.05$ ethanol/ TNBS-treated Nod2 - / - vs. Nod2 + / + by Student's $t$-test. (c, d) In vitro interferon $\gamma($ IFN- $\gamma$ ) and interleukin (IL)-17 production by unstimulated and antiCD3/CD28-stimulated lamina propria mononuclear cell (LPMC); data represent mean \pm s.e. of pooled values derived from three different experiments in which five mice per group were evaluated. Data were tested for statistical significance using one-way analysis of variance with Bonferroni's multiple comparison test.

regulatory response. As shown in Figure 5, panel a and Supplementary Figure 2, evidence that this was indeed the case came from studies showing that Nod $2-/-$ mice exhibited a greater increase in the percentage of LP CD $4+\mathrm{LAP}+\mathrm{T}$ cells among CD4 + LPMCs on the day of maximal permeability change and the relatively weak $\mathrm{LP} \mathrm{CD} 4+\mathrm{LAP}+\mathrm{T}$-cell response of $\mathrm{Nod} 2+1+$ mice was delayed. In both cases, however, the percentage of LP CD $4+\mathrm{LAP}+\mathrm{T}$ cells at day 3 did not differ from baseline levels of the untreated mice. Finally, as shown in Figure 5, panel b, Nod $-/-$ mice also mounted 
an earlier and higher in vivo TGF- $\beta$ production response than Nod $2+/+$ mice following i.r. ethanol administration, although responses in both groups of mice were increased under these circumstances.

As previously reported, ${ }^{11}$ and as shown in the flow cytometric study depicted in Supplementary Figure 2, the majority of LP CD4 + LAP + T cells on day 1 after ethanol challenge were Foxp3-negative. However, as also shown in this figure, the percentage of LP CD4 + LAP + Foxp3 $+\mathrm{T}$ cells among LPMC's also increased after ethanol administration at this time point. In addition, this increase was particularly evident $2-3$ days after ethanol challenge: (Nod $2-/-$ baseline: $11.71 \% \pm 0.74$ vs. Nod $2-/-$ day $3: 16.27 \% \pm 2.04$ mean \pm s.e., $P=0.01 ; \quad$ Nod $2+/+\quad$ baseline: $9.93 \% \pm 1.18$ vs. day $2: 16.03 \% \pm 2.85$ mean \pm s.e., $P=0.02$ ). Importantly, however, the increase in Foxp3 $+\mathrm{T}$ cells was equivalent in Nod2 $-/-$ and $\mathrm{Nod} 2+/+$ mice, indicating that this did not explain the differences in degree of ethanol-induced inflammation and cytokine response between these groups.

\section{The expansion of LP CD4 + LAP + T cells results in decreased severity of TNBS-colitis in Nod2 - I - compared with $\mathrm{Nod} 2+I+$ mice}

To obtain further evidence that the increased percentage of LP $\mathrm{CD} 4+\mathrm{LAP}+\mathrm{T}$ cells of Nod2 $-/-$ mice before or after ethanol treatment is preventing the development of colitis in Nod2 - / - mice we next conducted studies to determine if these cells affected the induction of TNBS-colitis, a colitis known to be modulated by LP CD $4+\mathrm{LAP}+\mathrm{T}$ cells and TGF$\beta{ }^{12}$ To ensure valid results we conducted studies in Nod2 - / mice and control Nod $2+1+$ mice that had been re-derived by embryo transfer into littermate foster mothers raised in the same SPF animal facility and in the same cage and then placed in separate cages before birth; thus, the mice had a similar origin and were reared in the same facility.

As shown in Figure 6, panels a and b, Nod2 $-/-$ mice administered i.r. TNBS (in 50\% ethanol) to induce TNBS-colitis developed less severe colitis than Nod $2+/+$ mice as shown by their lower weight loss and degree of colonic inflammation. In addition, as shown in Figure 6, analysis of anti-CD3/CD28induced LPMC cytokine production in vitro showed that while IFN $-\gamma$ production by LPMCs from Nod $2-/-$ mice was significantly higher than that of LPMCs from Nod $2+/+$ mice, IL-17 production by LPMCs from Nod $2-/-$ mice was significantly lower than that of LPMCs from Nod $2+/+$ mice and was equivalent to baseline (ethanol alone) IL-17 production. Taken together, these studies as well additional studies described below offer strong evidence that Nod2 - / mice, as compared with $\mathrm{Nod} 2+/+$ mice, are resistant to induction of an induced colitis, TNBS-colitis.

To determine whether the decreased severity of TNBS-colitis in Nod2 - / - mice was attributable to the induction of LP $\mathrm{CD} 4+\mathrm{LAP}+\mathrm{T}$ cells, we took advantage of our previous findings showing that LPMCs from mice treated with ethanol and thus with expanded LP CD4 + LAP + T-cell populations can protect mice from TNBS-colitis upon adoptive transfer to the latter when cells are transferred before but not after depletion of LAP $+\mathrm{T}$ cells. ${ }^{11}$ In studies along these lines, we assessed the severity of TNBS-colitis in Nod $2-/-$ and Nod $2+/+$ mice after adoptive transfer of LPMC from Nod2-/- and $\mathrm{Nod} 2+/+$ mice (respectively) that had been treated with ethanol (50\%) one day before cell harvest and transfer. As shown in Figure 7, panels a-d, recipients of LPMC from ethanol-treated mice exhibited amelioration of colitis as assessed by weight loss and histological evaluation of colonic inflammation; however, the level of amelioration was greater in $\mathrm{Nod} 2-/$ - recipients than in Nod $2+1+$ recipients possibly due to the fact that ethanoltreated LPMC from Nod $2-1$ - donors contains a higher level of $\mathrm{LAP}+\mathrm{T}$ cells. In contrast, as also shown in Figure 7, panels a-d, Nod2 - / - recipients of LPMCs from ethanol-treated mice that had be subjected to LAP + T-cell depletion (See Methods) exhibited more severe colitis than mice treated with TNBS alone and equivalent to that observed in Nod $2+1+$ treated with TNBS alone. Thus, the transferred LP CD4 + LAP + T-cell-depleted cell population derived from ethanol-treated mice contains cells that act as potent pro-inflammatory $\mathrm{T}$ cells in the TNBS-treated recipient mice (see further studies of cytokine production by these cells below). Finally, Nod $2+/+$ recipients of LPMCs from ethanol-treated mice that had been subjected to LAP + T-cell depletion exhibited the same level of colitis as mice treated with TNBS alone.

As shown in Figure 7, panels e-h, analysis of anti-CD3/ CD28-induced in vitro cytokine production, by equivalent numbers of cell per culture, revealed that amelioration of TNBS-colitis observed in Nod2-/- and Nod $2+/+$ recipients of LPMC (whether or not depleted of LAP + $\mathrm{T}$ cells) from ethanol-treated mice was not associated with changes in IFN- $\gamma$ production. However, in both recipient groups, whereas transfer of undepleted LPMC was associated with non-significant reductions in IL-17 production, transfer of depleted LPMC was associated with significant increases in IL-17 production. These data thus indicate that increased severity of TNBS-colitis in recipients of LPMC depleted of LAP + T cells correlates best with levels of IL-17 production per cell.

In complementary studies we performed adoptive transfer studies in which Nod $2+/+$ mice with TNBS-colitis were transferred LPMCs from ethanol-treated Nod2-/- mice rather than $\mathrm{Nod} 2+/+$ as in the experiments described above. As shown in Figure 8, transfer of LPMCs from Nod2-/mice, was associated with amelioration of colitis, but in this case the level of amelioration was somewhat greater than with transfer of LPMCs from Nod $2+/+$ mice. This is in keeping with the fact that ethanol treatment of Nod $2-/-$ mice led to the generation of a greater number of LP CD $4+\mathrm{LAP}+\mathrm{T}$ cells than ethanol treatment of Nod $2+/+$ mice (Figure 5) and that $\mathrm{Nod} 2+/+$ mice recipient of Nod 2 - / - LAP-depleted LPMC show a TNBS-colitis course comparable to the Nod $2+/+$ mice treated with TNBS alone.

Overall, then, these adoptive transfer studies of TNBS-colitis are compatible with the view that the LP CD4 + LAP + T-cell subpopulation generated by ethanol administration in 
Nod2 $-/-$ mice is capable of suppressing TNBS-colitis in both $\operatorname{Nod} 2-/-$ and $\operatorname{Nod} 2+/+$ mice and this suppression is greater than that obtained with a LP CD $4+\mathrm{LAP}+\mathrm{T}$-cell subpopulation generated by ethanol administration in $\operatorname{Nod} 2+/+$ mice.

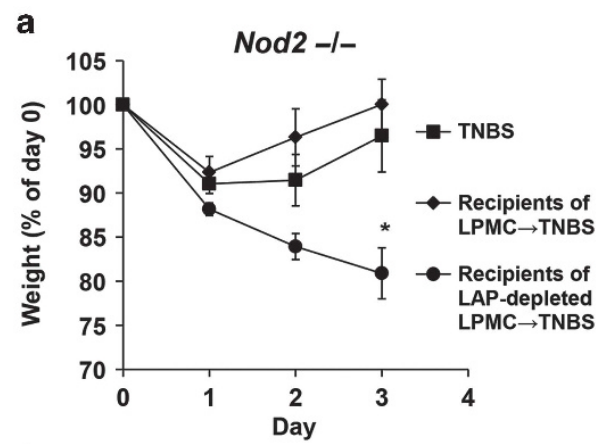

C

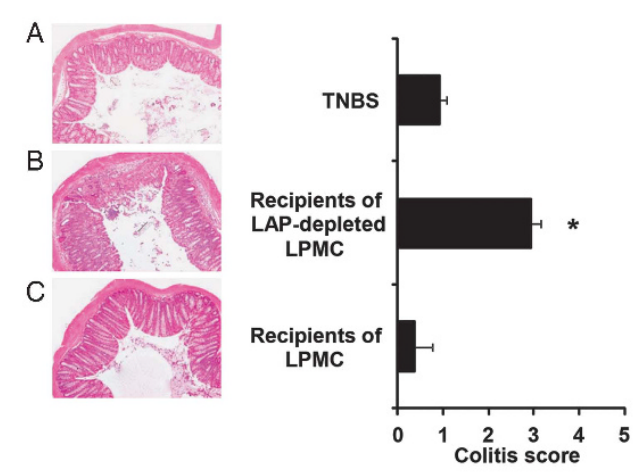

e

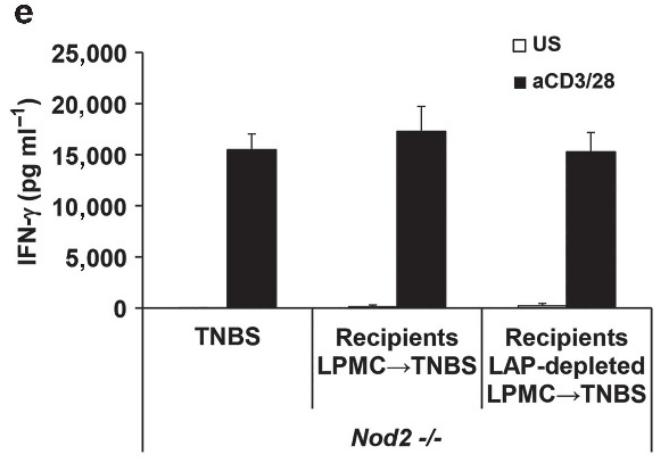

g

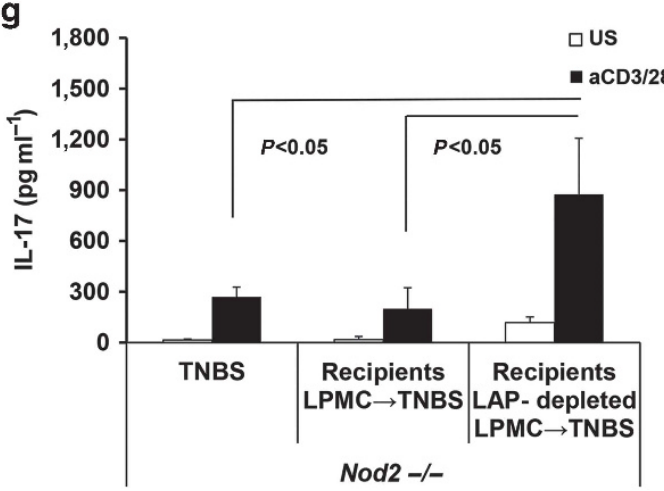

Influence of the endogenous Intestinal Microbiota on TNBS-colitis in Nod2-I- mice: co-housing studies In studies described above it was shown that the generation of LP CD $4+\mathrm{LAP}+$ regulatory cells was dependent on the presence of an intact intestinal microbiota. Thus, it seemed

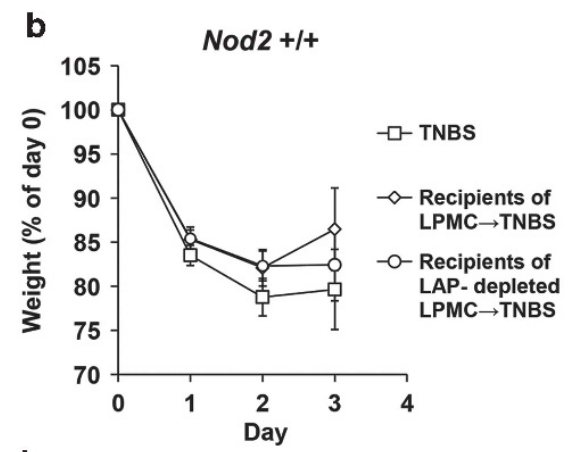

d
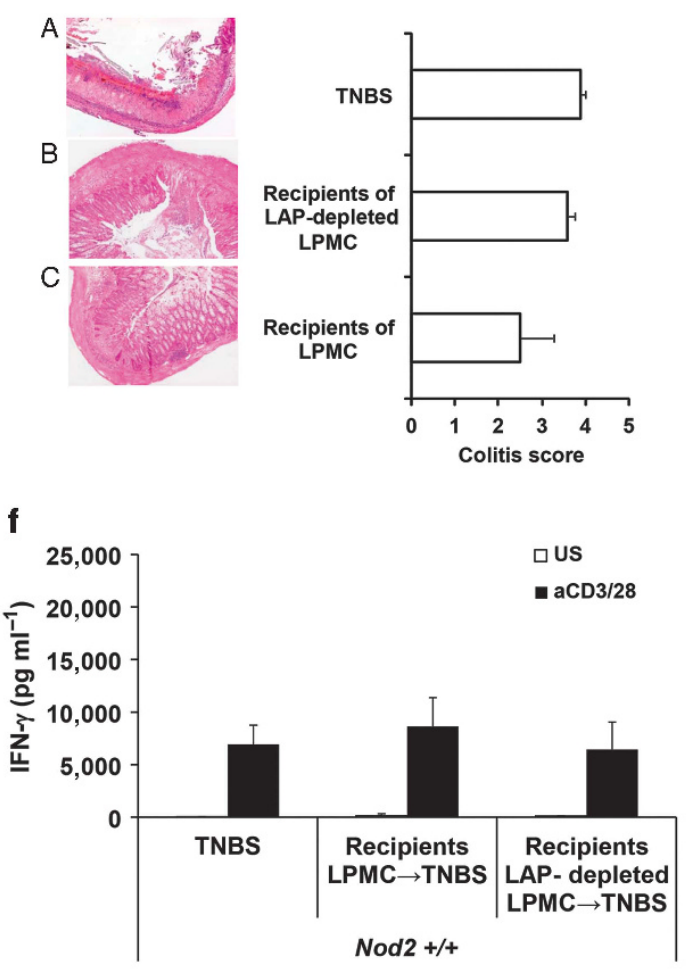

h

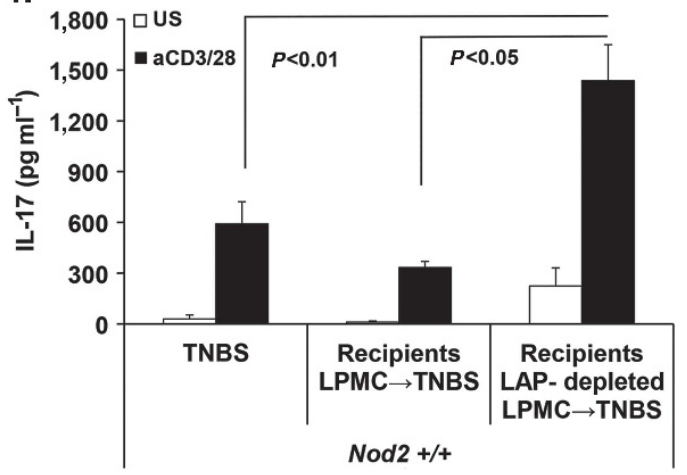


possible that Nod $2-/$ - mice harbor a changed microbiota that affects the development of regulatory cells in the Nod2deficient host. To examine this possibility, we conducted co-housing studies in which Nod $2-/-$ mice were maintained in the same cages with $\mathrm{Nod} 2+/+$ mice or maintained in separate cages at weaning for 4 weeks before induction of TNBScolitis. As shown in Figure 9, co-housed Nod2-/- mice exhibited more severe colitis and decreased LP CD $4+\mathrm{LAP}+\mathrm{T}$ cells than non-co-housed Nod2 $-/-$ mice. Reciprocal studies of Nod $2+/+$ showed that Nod $2+/+$ mice did not exhibit a change in baseline LP CD4+ LAP + cells (data not shown). Thus, the Nod2 - / - mice develop an intestinal microbiota that supports regulatory responses that contribute to the protection of mice from development of TNBS-colitis.

Dextran sulfate sodium (DSS) colitis in Nod2 - /- mice.

To determine if Nod2-deficient mice are protected from additional forms of experimental colitis we also assessed the severity of DSS-colitis in Nod $2-/-$ and Nod $2+/+$ mice. The mechanism of inflammation in DSS-colitis differs from that in TNBS-colitis in that it is dominated by a toxic effect of DSS on epithelial cells followed by massive stimulation of innate cells (macrophages) by commensal gut bacteria and activation of the NLRP3 inflammasome; ${ }^{13}$ thus, this colitis is less dependent on a T-cell response as is TNBS-colitis. As shown in Supplementary Figure 3, in agreement with previous reports, ${ }^{14,15}$ we observed that administration of low concentrations of DSS in drinking water led to more severe colitis in Nod $2-/$ - mice than in Nod $2+/+$ mice as shown by weight loss, myeloperoxidase activity and histology (the latter not shown). It was thus apparent that colitis dependent mainly on innate cell activation is under less regulation by LP CD4+ $\mathrm{LAP}+\mathrm{T}$ cells than is colitis dependent mainly on $\mathrm{T}$ cells.

\section{DISCUSSION}

In the present study, we investigated the factors accounting for the observation that mice with Nod2 deficiency do not exhibit significant spontaneous colonic inflammation despite the wellestablished role of NOD2 polymorphisms as risk factors in Crohn's disease. We found that Nod2-/- mice manifest increased colonic permeability and heightened LP IL-12p70 production associated with little or no change in downstream LP IFN- $\gamma$ production. The latter suggested the presence of a regulatory response and indeed the LP of Nod2-/- mice contained an increased subpopulation of LAP + regulatory $\mathrm{T}$ cells among the LP CD4 + cells that in subsequent studies was shown to be accentuated by i.r. ethanol administration and an accompanying increase mucosal permeability. We then showed that Nod2-/ - mice are resistant to the development of TNBS-colitis and that such resistance is likely due to the presence of the LP CD4 + LAP $+\mathrm{T}$ cells. In particular, we found that adoptive transfer of LP T cells from ethanol-treated mice enhanced protection from TNBS-colitis, whereas transfer of these cells following depletion of LAP $+\mathrm{T}$ cells not only abolished such protection, it led to a level of colitis observed in Nod $2+/+$ mice. Finally, we found that Nod $2-/-$ mice develop a microbiota that favors the development of LP $\mathrm{CD} 4+\mathrm{LAP}+$ regulatory cells and thus contributes to protection from induction of TNBS-colitis. These observations provide a strong rationale for why Nod2 deficiency does not itself lead to overt intestinal inflammation as well as why humans with NOD2 abnormalities require the presence of additional factors to manifest Crohn's disease.

Previous studies have provided evidence both in mice with Nod2 deficiency and in humans with NOD2 polymorphisms associated with Crohn's disease that NOD2 in myeloid cells functions as a negative regulator of TLR signaling and that such lack of "cross-regulation" of innate immune responses is a major reason why NOD2 abnormalities may underlie the excessive mucosal immune responses that lead to Crohn's disease. $^{7-9,16}$ In one of these previous studies as well as in the present study, this consequence of Nod2 deficiency could also explain the fact that Nod2 - / - mice exhibit high baseline (sub-inflammatory) mucosal cytokine production and increased activity of MLCK, a factor that is the likely cause of the increased gut permeability in these mice due to its effects on tight junctions. In addition, it could explain the dependence of these various abnormalities on the presence of the intestinal microbiota, the source of mucosal TLR responses and the increased capacity of LP CD11c + DCs from Nod $2-/$ - mice to induce LP CD4 + LAP + T cells compared with similar DCs from Nod $2+1+$ mice. On this basis the following sequence of events is a likely explanation for the increased LAP $+\mathrm{T}$ cells response in Nod2 - / - mice: (1) intestinal microbiota (via TLR stimulation) induce LP DCs in Nod2-/- mice to

Figure 7 Adoptive transfer of CD4 + LAP (latency-associated peptide) + T cells ameliorates severity of 2,4,6-trinitrobenzene sulfonic acid (TNBS)colitis. Mice were administered intrarectal ethanol/TNBS to induce TNBS-colitis alone or TNBS-colitis in adoptively transferred lamina propria mononuclear cells (LPMCs) or LAP + T-cell-depleted LPMCs mice. (a, b) Body weight changes; each point represents mean \pm s.e. weight at indicated times expressed as percentage of day 0 weight and derived from an experiment in which six mice per group were evaluated; ${ }^{*} P<0.05$ TNBS-colitis in nucleotide-binding oligomerization domain 2 (Nod2) - / - recipients of LAP + T-cell-depleted LPMC vs. recipients of LPMC and Nod2 - / - mice with TNBS-colitis alone; (c) histological appearance of representative colons of different groups of Nod2 - / - mice killed at day 3 after TNBS administration; hematoxylin and eosin $(\mathrm{H} \& \mathrm{E})$ staining, original magnification $\times 40$ : $(\mathrm{A})$ mild submucosal edema with mild inflammatory cell infiltration; $(B)$ moderate ulcerative colitis with moderate submucosal edema and inflammatory cell infiltration; (C) normal colonic architecture/patterns; Colitis score: ${ }^{\star} P<0.05$ TNBS-colitis in Nod2 - / - recipients of LAP-depleted LPMC vs. recipients of LPMC and Nod2 - / - mice with TNBS-colitis alone. (d) Histological appearance of representative colons of different groups of Nod2 + / + mice killed at day 3 after TNBS administration; H\&E staining, original magnification $\times$ 40: (A) severe, diffuse, transmural ulcerative colitis with severe, submucosal inflammatory cell infiltration also involving the smooth muscle and serosa layers; (B) marked ulcerative colitis with moderate submucosal edema and inflammatory cell infiltration; (C) moderate ulcerative colitis with moderate submucosal edema and inflammatory cell infiltration. (e-h) "In vitro" interferon $\gamma$ (IFN- $\gamma$ ) and interleukin (IL)-17 production by isolated LPMC. Data represent cumulative mean \pm s.e. from six mice per group. Data were tested for statistical significance using one-way analysis of variance with Bonferroni's multiple comparison test. 


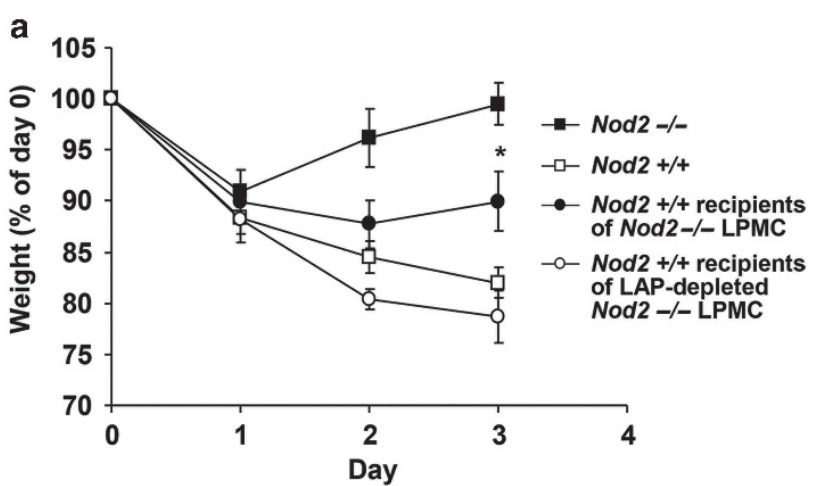

b

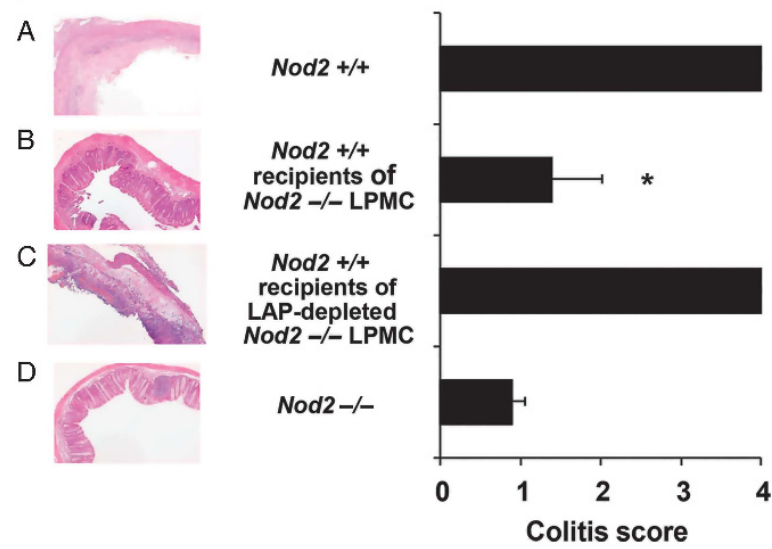

Figure 8 Mice were administered intrarectal ethanol/2,4,6trinitrobenzene sulfonic acid (TNBS) to induce TNBS-colitis in untreated nucleotide-binding oligomerization domain $2(\operatorname{Nod} 2)+/+$ mice or in $\mathrm{Nod} 2+/+$ mice adoptively transferred lamina propria mononuclear cells (LPMCs) or latency-associated peptide (LAP)-depleted LPMCs from ethanol-treated Nod2 - / - mice. (a) Body weight changes; each point represents mean \pm s.e. weight at indicated times expressed as percentage of initial weight; these data were from two experiments with eight mice per group except for LAP-depleted LPMC recipient group in which five mice were evaluated; ${ }^{*} P<0.05$; TNBS-colitis in Nod $2+/+$ recipients of Nod2 - / - LPMC vs. Nod2 +/+ mice with TNBS-colitis and Nod2 $+/+$ mice recipient of LAP-depleted Nod2 - / - LPMC. (b) Histological appearance of representative colons of different groups of mice killed at day 3 after TNBS administration; hematoxylin and eosin (H\&E staining), original magnification $\times 40$; (A) severe, diffuse, transmural ulcerative acute colitis with severe submucosal inflammatory infiltration deeply involving the smooth muscle and serosa layers; (B) moderate submucosal edema and moderate inflammatory infiltration; (C) severe, diffuse, transmural ulcerative acute colitis with severe submucosal inflammatory infiltration deeply involving the smooth muscle and serosa layers; (D) normal architecture/patterns with small lymphoid nodules. Colitis score; ${ }^{*} P<0.05$ TNBS-colitis in Nod $2+/+$ recipients of Nod2 - / - LPMC vs. Nod2 +/+ mice with TNBS-colitis and Nod $2+/+$ mice recipient of LAP-depleted Nod2 - / - LPMC. Data were tested for statistical significance using one-way analysis of variance with Bonferroni's multiple comparison test.

produce sub-inflammatory amounts of cytokines that stimulate activation of MLCK; (2) MLCK causes increased intestinal permeability and further exposure of LP DCs to microbiota TLR stimulation; (3) the DCs so-activated induce LP CD4+ LAP + regulatory cells. An additional factor bearing on the development of regulatory cells in Nod2-/ - mice is that, as discussed in greater detail below, the Nod2 deficiency in Nod2- / - mice leads to the development of an intestinal microbiota with an increased capacity to induce the development of regulatory cells. Thus, the effects of Nod 2 deficiency on intestinal permeability is abetted by its effects on the intestinal microbiota.

The above analysis of the generation of regulatory cells in Nod2-deficient mice is compatible with the somewhat unexpected view that the consequences of Nod2 dysfunction are twofold. On the one hand, it leads to a tendency to mount increased TLR-induced responses that favor the development of inflammation and thus explain its role as a risk factor for Crohn's disease. On the other hand, as shown in these studies, it also leads to an increased TLR-induced regulatory response that establishes a resistance to inflammation. Which of these tendencies prevail is likely to depend on the nature, strength and persistence of the inflammatory stimulus. When the stimulus is relatively weak and self-limited as in the present study of TNBS-colitis in C57BL/6 mice the regulatory response prevails and one sees protection from colitis; in contrast, when, in the same mice, the stimulus is strong as in DSS-colitis and accompanied by massive breakdown of the epithelial barrier leading to invasion of the mucosa by commensal organisms, the inflammatory response prevails and one sees more inflammation. These different inflammatory conditions might model different environmental triggers of Crohn's disease and thus explain disease initiation and recrudescence in patients with NOD2 abnormalities.

$\mathrm{LP} \mathrm{CD} 4+\mathrm{LAP}+\mathrm{T}$ cells are a unique regulatory cell population characterized by the presence of cell surface (LAPassociated) TGF- $\beta$ and the absence of Foxp 3 expression. As suggested by our previous observations in both mice and humans, this cell population tends to have a mucosal location and a size that is largely influenced by the prevailing microbiota. ${ }^{11,12,17}$ Thus, we showed in a murine model of experimental colitis ${ }^{12}$ and in humans with ileal-pouch-anal anastomosis for ulcerative colitis, ${ }^{17}$ that oral administration of a mixture of probiotics was able to expand LP CD4 $+\mathrm{LAP}+\mathrm{T}$ cells, while treatment of mice with oral antibiotics prevents their expansion. ${ }^{11}$ The regulatory activity of LP CD $4+\mathrm{LAP}+$ $\mathrm{T}$ cells has been highlighted in studies showing that they ameliorate colitis in both the cell transfer ${ }^{18}$ and TNBS-colitis models ${ }^{11,12}$ and has, in addition, been recently confirmed in a model of murine asthmatic lung inflammation. ${ }^{19}$ Finally, the regulatory activity of peripheral CD $4+\mathrm{LAP}+\mathrm{T}$ cells (possibly derived from the mucosal surface) has also been recently shown in humans. ${ }^{20}$ The results of these various studies of LP $\mathrm{CD} 4+\mathrm{LAP}+\mathrm{T}$ cells, taken in conjunction with those in the present study, establish that this cell population has an important role in maintaining immune homeostasis in the microbiota-rich environments of the mucosal surfaces.

In the present study, we observed a selective expansion of CD4 + LAP + Foxp3-T cells in Nod2 - / - mice as compared with $\mathrm{Nod} 2+/+\mathrm{T}$ cells but no comparable expansion of the $\mathrm{CD} 4+$ Foxp3 + cell population. However, the latter population did expand equally in Nod $2-/-$ and $\mathrm{Nod} 2+/+$ mice in response to a transient increase in intestinal permeability induced by i.r. ethanol administration. These results emphasize 

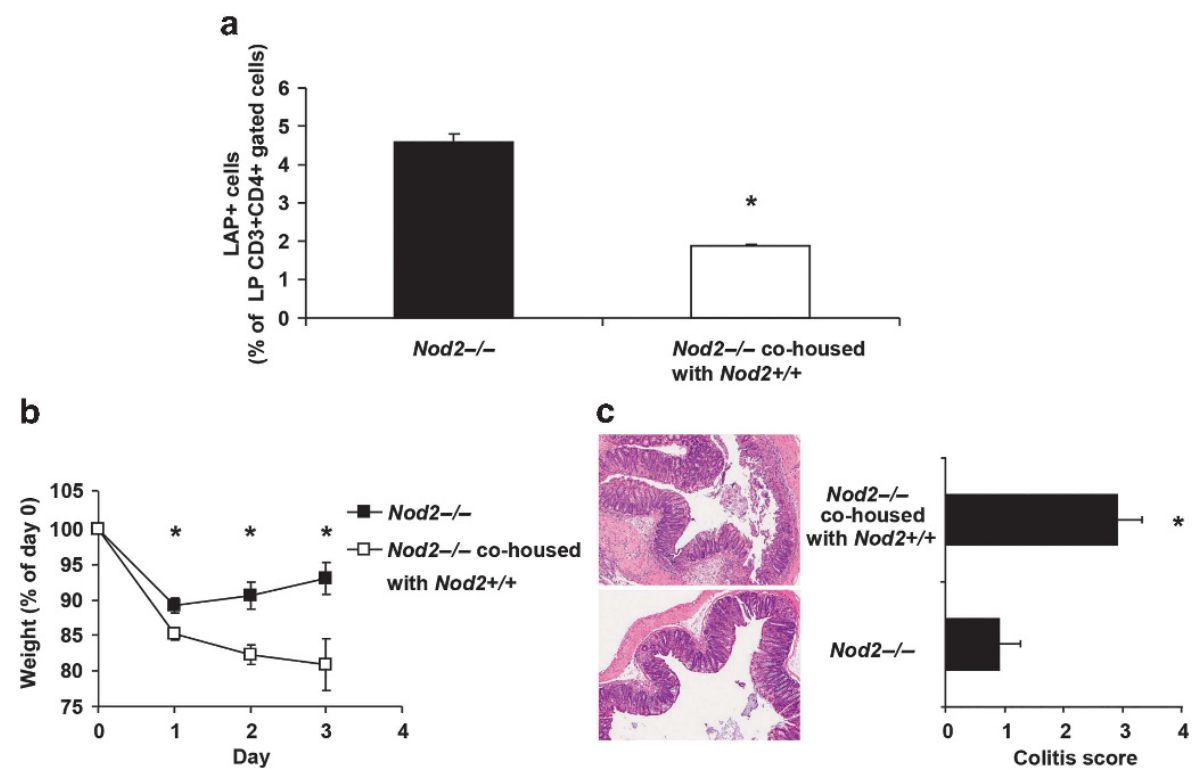

Figure 9 Nucleotide-binding oligomerization domain 2 (Nod2) $-/$ - mice were either single-housed $(n=5)$ or co-housed at a 1:1 ratio with Nod2 $+/+$ mice $(n=5)$ on the same diet for a 4-week period. (a) \% Of LP (lamina propria) CD4 + LAP (latency-associated peptide) + cells in the single-housed or cohoused mice. ${ }^{*} P<0.05$ by Student's $t$-test. (b) Body weight changes; each point represents mean \pm s.e. weight at indicated times expressed as percentage of day 0 weight. ${ }^{*} P<0.05$ by Student's $t$-test. (c) Histological appearance of representative colons of mice killed at day 3 after 2,4,6trinitrobenzene sulfonic acid (TNBS) administration; hematoxylin and eosin (H\&E) staining, original magnification $\times 40$. Colitis score: ${ }^{*} P<0.05$ by Student's $t$-test.

that $\mathrm{CD} 4+$ Foxp3 + cells are distinct from $\mathrm{CD} 4+\mathrm{LAP}+$ T-cell population in this context and do not underlie the intestinal regulatory response associated with Nod2 deficiency. Nevertheless, these two cell populations are likely to be related on some level given the fact that membrane-bound TGF- $\beta$ bound to LAP is present on both types of cells and, as such, is likely to be involved in their respective suppressive activities. ${ }^{21}$ In addition, it has recently been shown that Foxp3 induces surface TGF- $\beta / \mathrm{LAP}$ expression and that TGF- $\beta$ induces TGF$\beta /$ LAP expression in Foxp3- cells. ${ }^{22}$ This, plus the well known fact that TGF- $\beta$ induces Foxp3, suggests a broad (if as yet incompletely defined) inter-relation between TGF- $\beta$, Foxp3 and surface TGF- $\beta /$ LAP expression. This proposed interrelationship, however, does not necessarily imply that $\mathrm{CD} 4+$ LAP + Foxp3-T cells and CD4 + Foxp3 + cells belong to the same cell lineage or share the same set of inductive factors. ${ }^{23}$ The latter is evident from the present study in that the induction of $\mathrm{CD} 4$ + LAP + Foxp3- cells appears to require special conditions that include the presence of high levels of TGF$\beta$ as well as DCs producing a possibly unique mixture of cytokines resulting from the strong TLR signaling present in the LP in Nod $2-1-$ mice. This view is at least partially supported by the observation that the kinetics of LP CD4 + LAP + Foxp3T-cell expansion observed in our study parallels the kinetics of TGF- $\beta$ production and from previous evidence that the in vivo generation of CD4 + LAP + Foxp3- cells is dependent on both TGF- $\beta$ and IL- $10 .^{19}$

As indicated above, in the present study we observed that Nod2-/ - mice exhibit less severe TNBS-colitis when compared with Nod $2+/+$ mice. This finding correlated with the fact that in adoptive transfer studies we showed that LPMC from Nod $2-/-$ mice donor mice had a greater capacity to ameliorate colitis than cells from Nod $2+/+$ donor mice. In addition, we showed that this effect reflected the greater number of LP CD $4+\mathrm{LAP}+\mathrm{T}$ cells in the Nod $2-/-$ cell population as deletion of these cells from the transferred cell population abolished the protective effect of cell transfer.

Thus, these transfer studies provided a mechanistic basis for the decreased severity of colitis in Nod2 - / - mice. Somewhat unexpectedly, on a per cell basis, the decreased inflammation of TNBS-colitis in Nod2 - / - mice was associated with decreased LPMC IL-17 and increased IFN- $\gamma$ production as compared with Nod $2+/+$ mice. Furthermore, Nod $2-/$ - recipients of adoptively transferred LAP + T-cell-depleted LPMC exhibited a cytokine response comparable to that of $\mathrm{Nod} 2+1+$ mice that was characterized by increased LPMC production of IL-17 but no change in IFN- $\gamma$ production. These data suggesting that IL-17 production has a more important role in the pathogenesis of TNBS-colitis than IFN- $\gamma$ in C57BL/6 mice are in agreement with a previous report wherein it was shown that while such colitis was marked by increased in vivo IL-17 and IFN- $\gamma$ production, less severe colitis was observed in IL-17Rdeficient mice despite the presence of increased IFN- $\gamma$ tissue levels. ${ }^{24}$ In addition, the increase in IL-17 production in recipients of $\mathrm{CD} 4+\mathrm{LAP}+$-depleted cells suggests that the $\mathrm{CD} 4+\mathrm{LAP}+\mathrm{T}$ cells might be acting, in part, via regulation of Th17 cells.

The decreased severity of TNBS-colitis in Nod2 - / - mice observed in our study is in contrast with previous studies in which an increased severity of colitis in Nod2-/- mice compared with $\mathrm{Nod} 2+/+$ mice was reported. ${ }^{8,25}$ This may have been due to differences in the intestinal microbiota 
acquired by mice in the various mouse-housing facilities utilized ase it is known that at least IL-17 production can be influenced by nature of the resident microbiota. ${ }^{26}$ Thus, it is possible that Nod2-deficient mice is various facilities acquire somewhat unique sets of microbiota more or less capable of inducing pro-inflammatory effector cells and anti-inflammatory regulatory $\mathrm{T}$ cells that then serve as environmental stimuli leading to different Nod2-deficiency outcomes as discussed above. Also bearing on this issue are recent studies that have shown that changes in the intestinal microbiota are associated with Nod2 deficiency. ${ }^{27,28}$ Indeed, our studies corroborate this finding in that we have found in co-housing studies that Nod2 - / - mice reared together with Nod $2+/+$ mice postweaning developed less LP CD4 + LAP + cells and more severe TNBS-colitis than Nod $2-/-$ mice reared independently. It was thus evident that the microbiota in Nod2 $-/-$ mice was distinct from that in Nod $2+1+$ mice in that it had a greater capacity to induce regulatory cells. This implies that the Nod2 deficiency in Nod2 - / - mice operates in two ways to favor regulatory cell development: (1) it leads to permeability changes that increase exposure of DCs to factors such as TLR ligands that induce such development and (2) it creates a milieu that permits changes in the microbiota that support such development. At this point, the nature of these latter changes are quite undefined although we would speculate that they involve selective expansion of organisms similar to previously described inducers of regulatory cells such as members of the Clostridium bacterial family. ${ }^{29}$ Delineation of this possibility is clearly an area of potentially fruitful future work.

In summary, data from the present study suggest that the increased intestinal permeability observed in Nod2 - / - mice is associated with an increased homeostatic regulatory response mediated by LP CD $4+\mathrm{LAP}+\mathrm{T}$ cells. They thus suggest that factors influencing this regulatory response (or possibly overriding this response) might be necessary to induce a full-blown inflammation in the presence of Nod2 deficiency. This conclusion is relevant to IBD in humans where increased intestinal permeability ${ }^{30,31}$ and an altered microbiome ${ }^{32,33}$ have been reported to be associated with NOD2 polymorphisms in the absence of inflammation.

\section{METHODS}

Mice. Nod $2+/+$ and Nod $2-/$ - mice on C57BL/6 background were kindly donated by Peter J Murray, Department of Infectious Diseases and the Department of Immunology, St Jude Children's Research Hospital, Memphis, Tennessee, USA. ${ }^{34}$ At the Istituto Superiore di Sanità Nod $2-/-$ mice and control $\operatorname{Nod} 2+/+$ mice had been re-derived by embryo transfer into littermate foster mothers raised in the same SPF animal facility and in the same cage. Nod $2-/-$ and $\operatorname{Nod} 2+/+$ mice were then maintained in SPF conditions in the animal facility at the Istituto Superiore di Sanità, Roma, Italy. All mice were housed with free access to food and water. Pathogen-free conditions were monitored every 6 months in accordance with the full set of FELASA high standards recommendations. All animal studies were approved by the Animal Care and Use Committee of the Istituto Superiore di Sanità.

Study design. All studies were performed in 7-8-week-old male mice. In the studies of ethanol effects on regulatory T-cell expression, mice were administered $50 \%$ ethanol, via a $3.5-\mathrm{F}$ catheter inserted into the rectum as previously described; ${ }^{11}$ subsequently, mouse weights and histological appearance of the colonic tissue was determined at various time points. In studies on the effects of MLCK inhibition, mice were administered intraperitoneal injections ML-7 $\left(2 \mathrm{mg} \mathrm{kg}^{-1}\right.$; SigmaAldrich, Milan, Italy), a MLCK inhibitor, twice daily for 4 days. ${ }^{9}$ In studies on the effect of antibiotic administration, mice were administered ampicillin (Pfizer Italia srl, Latina, Italy) $200 \mu \mathrm{g}$ per $100 \mu \mathrm{l}$ distilled water by oral gavage for 14-21 days; control mice received water only. In studies of TNBS-colitis, mice were administered TNBS $3.5 \mathrm{mg}$ (Sigma-Aldrich) delivered in $150 \mu \mathrm{l}$ of $50 \%$ ethanol per rectum as previously described. ${ }^{11}$

Intestinal permeability studies. Untreated mice or ML-7 treated mice were administered with a dose of $2 \mathrm{mg}$ per $10 \mathrm{~g}$ body weight of FITCconjugated dextran dissolved in purified water $(4000 \mathrm{mw}$, SigmaAldrich) per rectum. Whole blood was obtained via eye bleed one hour after FITC-dextran administration. Blood samples were centrifuged at 3000 r.p.m. to obtain sera; the latter was then analyzed by fluorometry using a Victor3 $(485 / 535 \mathrm{~nm}$, gain 1,480$)$ plate reader (Perkin-Elmer, Waltham, MA). Mice administered 50\% ethanol per rectum were subsequently administered daily doses of FITC-conjugated dextran dissolved in purified water per rectum and whole blood was obtained via eye bleed $1 \mathrm{~h}$ after FITC-dextran administration.

Adoptive transfer of LPMC. LPMC were isolated from colons of $\operatorname{Nod} 2+/+$ and Nod $2-/$ - ethanol-treated mice 1 day after ethanol administration. LPMC $\left(4.0 \times 10^{5}\right)$ were then injected i.v. into the tail vein of $\mathrm{Nod} 2+/+$ and $\mathrm{Nod} 2-/-$ mice, respectively. In some experiments, additional mice of each group were administered LPMC depleted of LAP + T cells by cell sorting after staining cells with anti-mouse CD4-PerCP (RM4-5 BD-Pharmingen, St José, CA) and biotinylated goat anti-LAP (R\&D Systems, Minneapolis, MN)-streptavidin-APC using a FACS-ARIA. TNBS $3.5 \mathrm{mg}$ was administered per rectum to the recipient mice 5 days after the LPMC transfer as previously described. ${ }^{11}$ The occurrence and course of colitis in the different groups of animals was evaluated as described in the text.

Histological assessment of colitis. Tissues removed from mice at indicated times of death were fixed in $10 \%$ neutral buffered formalin solution (Sigma-Aldrich) and then embedded in paraffin, cut into tissue sections and stained with hematoxylin and eosin. Stained sections were examined for evidence of colitis by a pathologist (ISTOVET di Luca Crippa and C. S.A.S., Besana in Brianza (MB), Italy) and scored using previously reported criteria. ${ }^{11}$

Protein extraction. Mouse colonic specimens were rinsed with cold phosphate buffer solution, blotted dry and immediately frozen in liquid nitrogen for protein extraction.Total protein extracts were prepared as previously described. ${ }^{35}$ Briefly, snap frozen mucosal samples were homogenized in buffer containing $10 \mathrm{~mm}$ Hepes $(\mathrm{pH}$ 7.9), $60 \mathrm{~mm} \mathrm{KCl}, 1 \mathrm{~mm}$ ethylenediaminetetraacetic acid and $0.2 \%$ nonidet, supplemented with $1 \mathrm{~mm}$ dithiothreitol, $10 \mu \mathrm{g} \mathrm{ml}^{-1}$ aprotinin, $10 \mu \mathrm{g} \mathrm{ml}^{-1}$ leupeptin and $1 \mathrm{~mm}$ phenylmethanesulphonyl fluoride, $1 \mathrm{~mm}$ sodium orthovanadate, $1 \mathrm{~mm}$ sodium fluoride (all reagents were from Sigma, Milan, Italy).

Isolation and Culture of LP Mononuclear Cells (LPMC). LPMC were isolated from freshly obtained colonic specimens using a modification of the method described by van der Heijden and Stok. ${ }^{36}$ Isolated LPMC were cultured at $1 \times 10^{6}$ cells per $\mathrm{ml}$ in 48 -well plates (Costar, Corning Incorporated life sciences, Tewksbury, MA) coated or uncoated with murine anti-CD3e antibody (clone 145-2C11; BD-Pharmingen) and containing X-Vivo medium (Biowhittaker, Walkersville, MD) supplemented with $1 \mu \mathrm{g} \mathrm{ml}^{-1}$ of soluble anti-CD28 antibody (clone 37.51; BD-Pharmingen), as previously described. ${ }^{11}$ After $48 \mathrm{~h}$ culture, supernatants were harvested and stored at $-80^{\circ} \mathrm{C}$ for future enzymelinked immunosorbent assay (ELISA). 
In vitro Stimulation of CD4 + LP T Cells by CD11c + LP DCs. LPMC cells obtained from untreated mice were subjected to magnetic bead sorting using CD11c microbeads or a CD4 + T-cell Isolation Kit II (Miltenyi Biotech, Auburn, CA) following the manufacturer's instructions. The purity of LPMC CD11c + and CD4 + cells were $>90 \%$ and $>95 \%$, respectively, as assessed by immunofluorescence staining. In all, $2 \times 10^{5}$ per $\mathrm{ml}$ of the CD $4+$ cells obtained from Nod $2-/$ - or Nod $2+/+$ untreated mice were co-cultured with $1 \times 10^{5}$ per ml CD11c + cells obtained from Nod $2-/-$ or Nod $2+/$ + untreated mice in the presence or in the absence of $500 \mathrm{ng} \mathrm{ml}^{-1}$ Pam3CSK4 (InvivoGen, San Diego, CA). At the end of $48 \mathrm{~h}$ culture, the cells were collected, washed and stained for LAP expression by flow cytometry.

LPMC immunofluorescence staining. Freshly isolated and washed LPMC were subjected to Fc block with anti-CD16/CD32 (BDPharmingen) and then stained with biotinylated goat anti-LAP (R\&D Systems) or biotinylated normal goat IgG (R\&D Systems).

After $30^{\prime}$ incubation, cells were washed and incubated with streptavidin-APC. The cells were then incubated with PerCP-antimouse-CD3 (145-2C11, BD-Pharmingen) and APC-Cy7 anti-mouse CD4 (GK1.5, BD-Pharmingen), for an additional 30'. In some experiments, intracellular Foxp3 expression was evaluated using APC-anti-mouse/rat Foxp3 staining set (eBioscience, San Diego, CA) following the manufacturer's protocol. The cells were then washed twice, and the percentage of fluorescent cells was quantified using a FACS-Aria (BD Biosciences, Buccinasco, Milan, Italy).

ELISA. Cytokine concentrations in protein extracts and cell supernatants were determined by commercially available specific ELISA kits. IFN- $\gamma$ : BD-OptEIA Set Mouse (BD Bioscience); IL-12p70: Quantikine ELISA (R\&D Systems); IL-17: Quantikine ELISA (R\&D Systems), IL-17: BD-OptEIA Set Mouse (BD Bioscience); mouse/rat/ porcine/canine TGF- $\beta 1$ quantikine (R\&D systems) per the manufacturer's recommendations).

Statistical analysis. Assessment of statistical differences was determined by Student's $t$-test or by one-way analysis of variance with Bonferroni's multiple comparison test when appropriate.

SUPPLEMENTARY MATERIAL is linked to the online version of the paper at http://www.nature.com/mi

\section{ACKNOWLEDGEMENTS}

We thank Nazzareno Di Carlo (Immune-Mediated Diseases Section, Department of Infectious, Parasitic, and Immune-Mediated Diseases, Istituto Superiore di Sanità, Rome, Italy) for animal care and technical assistance. This work was supported in part by Grant ISS/NIH: FASC. 11 US/27 "Regulation of cell responses of components of the commensal microflora in Nod2-deficient mice" from the Italian Ministry of Health.

\section{DISCLOSURE}

The authors declared no conflict of interest.

c 2014 Society for Mucosal Immunology

\section{REFERENCES}

1. Strober, W., Murray, P.J., Kitani, A. \& Watanabe, T. Signalling pathways and molecular interactions of NOD1 and NOD2. Nat. Rev. Immunol. 26, 9-20 (2006).

2. Inohara, N., Chamaillard, M., McDonald, C. \& Nunez, G. NOD-LRR proteins: role in host-microbial interactions and inflammatory disease. Annu. Rev. Biochem. 74, 355-383 (2005).

3. Hugot, J.P. et al. Association of NOD2 leucine-rich repeat variants with susceptibility to Crohn's disease. Nature 411, 599-603 (2001).

4. Ogura, Y. etal. A frameshift mutation in NOD2 associated with susceptibility to Crohn's disease. Nature 411, 603-606 (2001).
5. Kobayashi, K.S. et al. Nod2-dependent regulation of innate and adaptive immunity in the intestinal tract. Science $\mathbf{3 0 7}, \mathbf{7 3 1 - 7 3 4}$ (2005).

6. Maeda, S. et al. Nod2 mutation in Crohn's disease potentiates NF-kappaB activity and IL-1beta processing. Science 307, 734-738 (2005).

7. Watanabe, T. et al. NOD2 is a negative regulator of Toll-like receptor 2-mediated Thelper type 1 responses. Nat. Immunol 5, 800-808 (2004).

8. Barreau, F. et al. CARD15/NOD2 is required for Peyer's patches homeostasis in mice. PLoS ONE 2, e523 (2007).

9. Barreau, F. et al. Nod2 regulates the host response towards microflora by modulating T-cell function and epithelial permeability in mouse Peyer's patches. Gut 59, 207-217 (2010).

10. Strober, W. \& Watanabe, T. NOD2, an intracellular innate immune sensor involved in host defense and Crohn's disease. Mucosal Immunol 4, 484-495 (2011).

11. Boirivant, M. et al. A transient breach in the epithelial barrier leads to regulatory T-cell generation and resistance to experimental colitis. Gastroenterology 135, 1612-1623 (2008).

12. Di Giacinto, C., Marinaro, M., Sanchez, M., Strober, W. \& Boirivant, M. Probiotics ameliorate recurrent Th1-mediated murine colitis by inducing IL-10 and IL-10-dependent TGF- $\beta$-bearing regulatory cells. J. Immunol 174, 3237-3246 (2005).

13. Bauer, C. et al. Colitis induced in mice with dextran sulfate sodium (DSS) is mediated by the NLRP3 inflammasome. Gut 59, 1192-1199 (2010).

14. Dalmasso, G. et al. The PepT1-NOD2 signaling pathway aggravates induced colitis in mice. Gastroenterology 141, 1334-1345 (2011).

15. Couturier-Maillard, A. et al. NOD2-mediated dysbiosis predisposes mice to transmissible colitis and colorectal cancer. J Clin Invest 123, 700-711 (2013).

16. Hedl, M., Li, J., Cho, J.H. \& Abraham, C. Chronic stimulation of Nod2 mediates tolerance to bacterial products. Proc. Natl Acad. Sci 104, 19440-19445 (2007).

17. Pronio, A. et al. Probiotic administration in patients with ileal pouch-anal anastomosis for ulcerative colitis is associated with expansion of mucosal regulatory cells. Inflamm. Bowel Dis. 14, 662-668 (2008).

18. Oida, T. et al. CD4 + CD25- Tcells that express latency-associated peptide on the surface suppress CD $4+$ CD45Rb high-induced colitis by a TGF- $\beta$ dependent mechanism. J. Immunol. 170, 2516-2522 (2003).

19. Duan, W., So, T., Mehta, A.K., Choi, H. \& Croft, M. Inducible CD4+ $\mathrm{LAP}+$ Foxp3 + regulatory $\mathrm{T}$ cells suppress allergic inflammation. J. Immunol. 187, 6499-6507 (2011).

20. Gandhi, R., Farez, M.F., Wang, Y., Kozoriz, D., Quintana, F.J. \& Weiner, H.L. Cutting edge: human latency-associated peptide $+T$ cells: a novel regulatory T cell subset. J. Immunol. 184, 4620-4624 (2010).

21. Nakamura, K., Kitani, A. \& Strober, W. Cell contact-dependent immunosuppression by CD $4+\mathrm{CD} 25+$ regulatory $T$ cells is mediated by cell surface-bound transforming growth factor $\beta$. J. Exp. Med 194, 629-644 (2001).

22. Oida, T. \& Weiner, H.,L. TGF- $\beta$ induces surface LAP expression on murine CD4 T cells independent of Foxp3 induction. PLOS ONE 5, e15523 (2010).

23. Iliev, I.D., Mileti, E., Matteoli, G., Chieppa, M. \& Rescigno, M. Intestinal epithelial cells promote colitis-protective regulatory T-cell differentiation through dendritic cell conditioning. Mucosal Immunol. 2, 340-350 (2009).

24. Zhang, Z., Zheng, M., Bindas, J., Schwarzenberger, P. \& Kolls, J.K. Critical role of IL-17 receptor signaling in acute TNBS-induced colitis. Inflamm. Bowel Dis. 12, 382-388 (2006).

25. Penack, O. et al. NOD2 regulates hematopoietic cell function during graftversus-host disease. J. Exp. Med. 206, 2101-2110 (2009).

26. Ivanov, I.I. et al. Induction of Intestinal Th17 Cells by segmented filamentous bacteria. Cell 139, 485-498 (2009).

27. Rehman, A. et al. Nod2 is essential for temporal development of intestinal microbial communities. Gut 60, 1354-1362 (2011).

28. Mondot, S. et al. Altered gut microbiota composition in immune-impaired Nod2 - / - mice. Gut 61, 634-635 (2012).

29. Atarashi, K. et al. Induction of colonic regulatory T cells by indigenous Clostridium species. Science 331, 337-341 (2011).

30. Buhner, S. et al. Genetic basis for increased intestinal permeability in families with Crohn's disease: role of CARD15 3020insC mutation? Gut 55, 342-347 (2006). 


\section{ARTICLES}

31. D'Incà, R. et al. Increased intestinal permeability and NOD2 variants in familial and sporadic Crohn's disease. Aliment. Pharmacol. Ther. 23, 1455-1461 (2006).

32. Frank, D.N. et al. Disease phenotype and genotype are associated with shifts in intestinal-associated microbiota in inflammatory bowel diseases. Inflamm. Bowel Dis. 17, 179-184 (2011).

33. Li, E. et al. Inflammatory bowel diseases phenotype, C. difficile and NOD2 genotype are associated with shifts in human ileum associated microbial composition. PLOS ONE 7, e26284 (2012).
34. Pauleau, A.L. \& Murray, P.J. Role of Nod2 in the response of macrophages to Toll-like receptor agonists. Mol. Cell Biol. 23, 7531-7539 (2003).

35. Boirivant, $M$. et al. Inhibition of Smad7 with a specific antisense oligonucleotide facilitates TGF-beta1-mediated suppression of colitis. Gastroenterology 131, 1786-1798 (2006).

36. Van der Heijden, P.J. \& Stok, W. Improved procedure for the isolation of functionally active lymphoid cells from the murine intestine. J. Immune Methods 103, 161-167 (1987). 